\title{
The Variable Wiener Index of Trees with Given Maximum Degree* $^{*}$
}

\author{
Zhen Jia, Ning Lin, Hongjie Gao, Zhilin Chen, Zhiyuan Wang and Fuyi Wei* \\ Department of Mathematics South China Agricultural University, Guangdong Province, China \\ "E-mail: weifuyi@126.com
}

\begin{abstract}
Wiener index proposed by Harold Wiener in 1947 is a very important topological index which is widely applied in chemical research. Gutman et al. extended the Wiener index to variable Wiener index. M. H. Liu et al. obtained the maximum variable Wiener index of trees with given maximum degree $\Delta \geq \frac{\mathbf{n}}{2}$.
\end{abstract} In this paper, we obtained the second largest variable Wiener index of trees with given maximum degree $\Delta \geq \frac{\mathrm{n}}{2}$ by using the method of control variables and computer programming.

Index Terms - variable Wiener index, tree, maximum degree

\section{Introduction}

Let graph $T(V, E)$ be a tree with $|V|=\mathrm{n}$, and $d(u)$, called the degree of the vertex $u$, be the number of the neighbors of vertex $u$ in $T(V, E) . \Delta(T)$ is denoted as the maximum degree of vertices in tree $T(V, E)$, and $T_{\mathrm{n}}^{\Delta}$ be the set of trees with maximum degree $\Delta$ and vertices $n$. As a general rule, let $K_{1, \mathrm{n}-1}, C_{n}, P_{n}$ be the star, cycle, and path of order $n$, respectively. A caterpillar is a tree denoted by $T\left(n, d ; n_{1}, n_{2}, \ldots, n_{d-1}\right)$ obtained from a path $v_{0} v_{1} \cdots v_{d}$ by attaching $n_{i}\left(n_{i} \geq 0\right)$ pendant vertices to vertex $v_{i}(i=1,2, \ldots, d-1)$.The caterpillar $T(n, n-\Delta+1 ; 0,0, \ldots, \Delta-2) \quad, \quad T(n, n-\Delta$; $1,0,0, \ldots, \Delta-2)$ and $T(n, n-\Delta+1 ; 0,0, \ldots, \Delta-2,0)$ denoted by $C(n, \Delta)$ (see Figure 1$), F(n, \Delta)$ (see Figure 2) and $H(n, \Delta)$ (see Figure 3$)$, respectively. When $\Delta=n-1$, it is the graph $K_{1, n-1}$. When $\Delta=n-2$, the graph is $C(n, \Delta)$. So we discuss the second largest variable Wiener index of trees with given maximum degree under the condition of $\Delta \leq n-3$. (see Figure right)

As early as in 1947[1], Harold Wiener used Wiener index denoted as $W(G)$ to calculate the boiling points of paraffin, and Wiener himself conceived $W(G)$ only for acyclic molecules, so the formula is followed as

$$
W(T)=\sum_{e} n_{1}(e) \cdot n_{2}(e)
$$

where $\mathrm{n}_{1}(e), n_{2}(e)$ are the number of vertices on the two sides of the edge $e$ respectively.
There are many research about Wiener index[2-3]. Nikolic et al.[4] put forward a modified Wiener index defined as

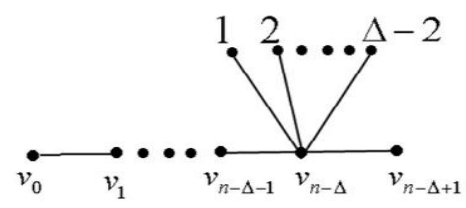

Fig. $1 \quad C(n, \Delta)$

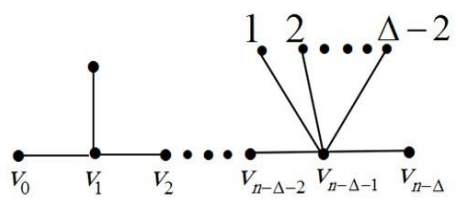

Fig. $2 F(n, \Delta)$

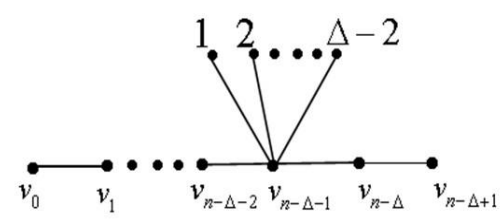

Fig. $3 H(n, \Delta)$

$$
{ }^{m} W(T)=\sum_{e}\left(n_{1}(e) \cdot n_{2}(e)\right)^{-1}
$$

Motivated by the analogy between equation (1) and (2), Gutman et al.[5] extended the definitions of $W(T)$ and ${ }^{m} W(T)$ to

$$
{ }^{m} W_{\lambda}(T)=\sum_{e}\left(n_{1}(e) \cdot n_{2}(e)\right)^{\lambda}
$$

${ }^{\mathrm{m}} W_{\lambda}(T)$ is defined as the variable modified Wiener index of $T$. This index introduce the parameter $\lambda$, there is research show that the optima value of $\lambda$ gives the smallest standard error of estimate in the structure-boiling point modelling of isomeric octane[6]. The relation between variable Wiener

* The work is supported by Training and plan projects of college students' innovation and pioneering in Guangdong Province(No.1056412059). 
index and internal molecular energy was also studied[7]. I. Gutman et al.[5] determined the path has the maximum variable Wiener index in trees when $\lambda>0$. M.H. Liu et al. ${ }^{[8]}$ obtained the $C(n, \Delta)$ has the maximum variable Wiener index of trees with given maximum degree $\Delta \geq \frac{n}{2}$.

In this paper, we obtain the second largest variable Wiener index of trees with given maximum degree $\Delta \geq \frac{\mathrm{n}}{2}$ by using the method of control variables and computer programming.

\section{The Second Largest Variable Wiener Index of Trees with Given Maximum Degree}

In this section, we use the method of control variables and computer programming to obtain the conclusion.

Lemma 2.1[8] If $T$ is a tree with $\Delta \geq \frac{n}{2}$ in $T_{n}^{\Delta}$, then ${ }^{m} W_{\lambda}(T) \leq{ }^{m} W_{\lambda}(C(n, \Delta))$ for $\lambda>0$, the equality holds if and only if $T=C(n, \Delta)$.

Lemma 2.2[8] If $T$ is a tree with $\Delta \geq \frac{n}{2}$ in $T_{\mathrm{n}}^{\Delta} \backslash\{C(n, \Delta), F(n, \Delta)$, $H(n, \Delta)\}$, then ${ }^{m} W_{\lambda}(T)<\max \left\{{ }^{m} W_{\lambda}(F(n, \Delta)),{ }^{m} W_{\lambda}(H(n, \Delta))\right\}$

for $\lambda>0$.

Lemma 2.3 (Intermediate value theorem) Let $f(x)$ be a continuous function on the interval $[a, b]$ and $f(a) \cdot f(b)<0$, then there is a real value $\xi \in(a, b)$, such that $f(\xi)=0$.

From the lemma 2.2, it is easy to know that the second largest variable Wiener index of trees is the larger one of $F(n, \Delta)$ and $H(n, \Delta)$ for $\lambda>0$. Now we define the function and the equation: $f(\lambda, n, \Delta)={ }^{m} W_{\lambda}(F(n, \Delta))-{ }^{m} W_{\lambda}(H(n, \Delta))$ and $f(\lambda, n, \Delta)=0$

If we find the root $\lambda$ is the only one of the equation, then the second largest variable Wiener index can be determined easy. By the bisection method we can compute the value of the root $\lambda$, and compare the value ${ }^{m} W_{\lambda}(F(n, \Delta))$ of $F$ and ${ }^{m} W_{\lambda}(H(n, \Delta))$ of $H$ for brevity, represented in the following table:

TABLE I Comparison the value of $\mathrm{F}$ and $\mathrm{H}$

\begin{tabular}{|c|c|c|c|}
\hline$\left(\Delta \geq \frac{n}{2}, n\right)$ & \multicolumn{2}{|c|}{$\begin{array}{c}\Delta=n-3 \text { and } n>13, \mathrm{o} \\
\mathrm{r} \\
\Delta=n-4 \text { and } \\
13<n \leq 112\end{array}$} & $\begin{array}{c}\Delta<n-4 \text { and } n>13, \\
\quad \text { or } \\
\Delta=n-4 \text { and } n>112\end{array}$ \\
\hline$\lambda(\lambda>0)$ & \multicolumn{2}{|c|}{ only one root $\lambda_{0}$} & no positive roots \\
\hline$f(\lambda, n, \Delta)$ & & & $f(\lambda, n, \Delta)>0$ \\
\hline \multirow{2}{*}{$\begin{array}{l}\text { The larger one } \\
\text { in } \boldsymbol{F}, \boldsymbol{H}\end{array}$} & $\lambda<\lambda_{0}$ & $\lambda>\lambda_{0}$ & \multirow{2}{*}{$F>H$} \\
\hline & $F<H$ & $F>H$ & \\
\hline
\end{tabular}

\section{Remark}

(1) When $\mathrm{n} \leq 13$, there is at most one root corresponding to the each variation of $\Delta$, it is very complex, so we don't represent this case.

(2) The root $\lambda$ is variable with the value $n, \Delta$. The research about the root $\lambda$ is discussed in other paper.

From the table, we can draw conclusions as follows:

Conclusion 1 When $n>13$ with $\frac{n}{2} \leq \Delta<n-4$, or $n>112$ with $\Delta=n-4, F(n, \Delta)$ has the second largest variable Wiener index of trees with given the maximum degree.

Conclusion 2 When $n>13$ with $\Delta=n-3$, or $13<n \leq 112$ with $\Delta=n-4, F(n, \Delta)$ has the second largest variable Wiener index of trees with given the maximum degree, where $\lambda$ is larger than the only one positive root of $f(\lambda, n, \Delta)=0$. In reverse, $H(n, \Delta)$ has the second larger variable Wiener index of trees with given the maximum degree, where $\lambda$ is smaller than the only one positive root of $f(\lambda, n, \Delta)=0$.

\section{Acknowledgment}

Support of the Training and plan projects of college students' innovation and pioneering in Guangdong Province (No. 1056412059) is gratefully acknowledged. We are also grateful to the referees for their valuable comments and corrections, which lead to an improvement of the original manuscript. Corresponding author: Weifuyi.

\section{References}

[1] H.Wiener. "Structral determination of paraffin boiling points," J. Am. Chem. Soc., vol. 69, pp. 17-20. 1947.

[2] M.V. Diudea and I. Gutman, "Wiener-Type Topological indices", Croat. Chem. Acta, vol.71, no. 1, pp. 21-51, 1998.

[3] I. Lukovits, "Wiener-type graph invariants, in QSPR/QSAR Studies by Molecular Descriptors (ed. MV Diudea)," Nova Science, Huntington, NY, pp. 31-38.

[4] S. Nikolic, N. Trinajstic and M. Randic, "Wiener index revisited," Chem. Phys. Lett., vol. 333, pp. 319-321, 2001.

[5] I. Gutman, D. Vukicevic and J. Zerovnik, "A class of modified Wiener indices," Croat. Chem. Acta, vol. 77, pp. 103-109, 2004.

[6] B. Lucic, A. Milicevic, S. Nikolic and N. Trinajstic, "On variable Wiener index, "Indian J. Chem., vol. 42A, pp. 1279-1282, 2003.

[7] I. Gutman, D. Vidovic, B. Furtula and I. G. Zenkevich, "Wiener-type indices and internal molecular energy," J. Serb. Chem. Soc., vol. 68, pp. 401-408, 2003.

[8] M.H. Liu and B.L. Liu, "On the variable Wiener indices of trees with given maximum degree," Math. Comput. Modelling, vol. 52, pp. 1651$1659,2010$. 